\title{
Multipactor in crossed rf fields on the cavity equator
}

\author{
Valery Shemelin* \\ Cornell Laboratory for Accelerator-based Sciences and Education (CLASSE), Ithaca, New York 14853, USA
}

(Received 10 September 2012; published 8 January 2013)

\begin{abstract}
Multipactor discharge in an accelerating superconducting elliptic cavity occurs usually near its equator. As simulations show, the dimensions of the trajectories of multipacting electrons are very small compared to the dimensions of a cavity. This feature gives a way for solving explicit equations of motion instead of cumbersome simulations. Electric and magnetic fields near the cavity equator are presented in a form of expansions up to the third power of coordinates. Comparisons with numerical calculations of fields made with the SLANS code for the TESLA cavity cells, as well as with the analytical solution for a spherical cavity, are done. These fields are used for solving the equations of motion of electrons in crossed rf fields near the equator. Based on the analysis of these equations, general features of multipacting in this area are obtained. Results are compared with simulations and experimental data. The experimental formulas for multipacting zones are explained and their dependence on the cavity geometries is shown. Because of small sizes of electron trajectories, the influence of the weld seams is taken into account. This suggests a possible explanation of multipacting in a cavity which was not found by simulations. The developed approach allows evaluation of multipacting in a cavity without its simulations but after an analysis of fields in the equatorial region. These fields can be computed by any code used for cavity calculation.
\end{abstract}

DOI: 10.1103/PhysRevSTAB.16.012002

PACS numbers: 52.80.Pi, 79.20.Hx, 02.60.-x, 85.25.- j

\section{INTRODUCTION}

Multipactor discharge in accelerating rf cavities remains to be a phenomenon that is difficult to predict quantitatively by a theory. Especially sensitive to multipacting are the superconducting cavities when their advantage, high quality factor, is suddenly spoiled by parasitic electron loading.

The change from a rectangular to circular and then to elliptical cross section of the equatorial region helps to alleviate the problem. However, even with the rounded shape of the equator as, for example, in the TESLA cavities, a multipactor appears sometimes and requires a certain time for processing. Our simulations show that resonant motions of electrons are possible in this cavity, but that particles have low impact energy $(32 \mathrm{eV})$ at which the secondary electron yield should be less than unity. Nevertheless, the rf processing is required to get rid of multipacting in this area.

Although simulations can help in many cases, they require special programs which are time consuming. However, they cannot give a clear hint on how to change the cavity shape to obviate multipacting.

On the other hand, the results of simulations can be helpful for further analysis of motion and prompt a

\footnotetext{
*vs65@cornell.edu
}

Published by the American Physical Society under the terms of the Creative Commons Attribution 3.0 License. Further distribution of this work must maintain attribution to the author(s) and the published article's title, journal citation, and DOI. possible way of better understanding the phenomenon. For example, let us consider the trajectory obtained in multipacting calculations for a cavity [1], Fig. 1. The calculations were performed with MULTIPAC [2]. One can see that the dimensions of the trajectory are very small compared with the cavity dimensions. It is clear that the magnetic field has as big an influence on the motion as the electric field. At the same time, one can assume that the amplitude of the magnetic field is nearly constant within such a small change of coordinates. These features will be used in the derivation of the equations of motion.

Of course, integration of equations of motion is performed in any simulation. But these are most general equations taking into account fields in the whole cavity. We see our task as minimizing the quantity of data required to compute the equations of motion. Ideally, data can be expressed in terms of geometrical parameters. The analytical presentation of the equations can give figures of merit that determine the phenomenon. For example, for multipacting in a flat gap, the value of $f d$, the frequency of oscillations times the gap length (or $\omega d$, where $\omega=2 \pi f$ ), defines limits of normalized voltage $\xi=e U / m \omega^{2} d^{2}$ across the gap where the discharge takes place [3]. It appears that, in the case of multipacting near the cavity equator, there are also two defining parameters. The first of them is a geometrical parameter depending on the curvature of the surface near the equator and some other geometrical details but not on the field amplitudes: $p=$ $\left(d E_{n} / d x\right) / \omega B_{0}$, where $E_{n}$ is the normal to the surface electric field at the area of multipacting, $B_{0}$ is the magnetic 
MultiPac 2.1 Electron Trajectory, $\mathrm{N}=20$
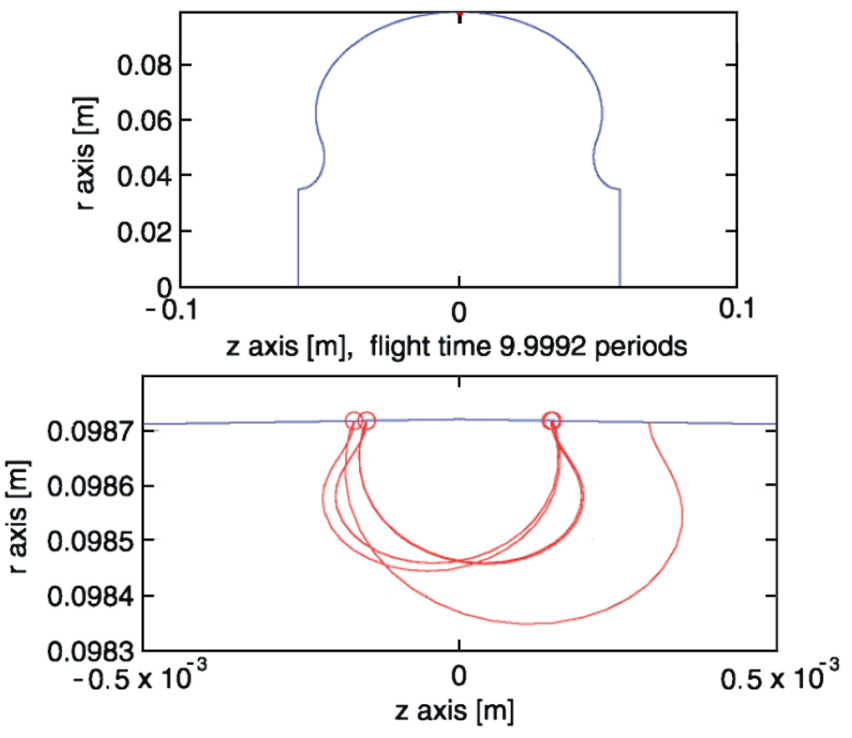

FIG. 1. Resonant electron orbits at a peak surface field level about $37 \mathrm{MV} / \mathrm{m}$.

field in this area, and the derivative is taken along the surface transversely to the magnetic field. A parameter proportional to the intensity of fields, or the magnetic parameter, is $M=e B_{0} / m \omega$. Here $e / m$ is the specific charge of the electron.

\section{FIELDS NEAR EQUATOR}

The shape of the cavity, Fig. 2, is assumed to consist of two elliptic arcs: one near the equator and another near the

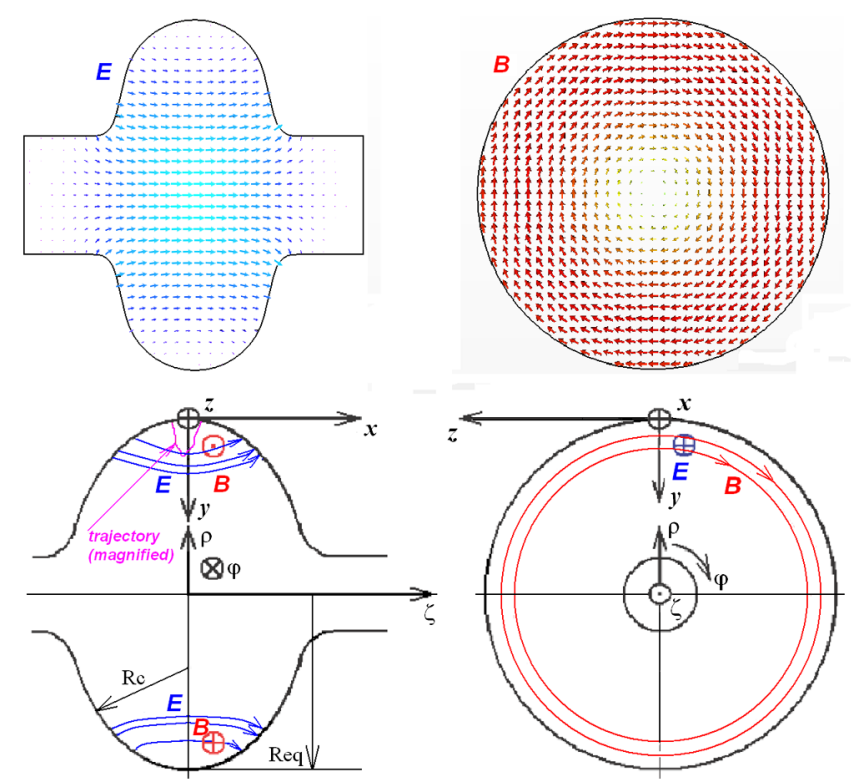

FIG. 2. Fields in an accelerating cavity as a whole (upper pictures) and the coordinate systems for calculation of multipacting near the equator. beam pipe. There can be a straight segment between these arcs.

In the upper row of this figure the electric $(E)$ and magnetic $(B)$ fields are shown for the cavity as a whole. The electric field on the cavity axis serves for acceleration of charged particles. Two-point multipacting usually occurs near the equator.

We will analyze the electric and magnetic fields in the immediate vicinity of the equator, so we can suppose that they depend first of all on the radius of curvature $R_{c}$ near the equator, not the lengths of the ellipses axes. The equatorial radius of the cavity is designated as $R_{\text {eq }}$. We describe fields near the cavity equator in the cylindrical system of coordinates: $\rho, \varphi, \zeta$. Let us present the fields in the form of series up to the third order of increments:

$$
\begin{aligned}
E_{\zeta}= & \omega B_{0}\left[\alpha_{0}\left(R_{\mathrm{eq}}-\rho\right)+\alpha_{1}\left(R_{\mathrm{eq}}-\rho\right)^{2}+\alpha_{2} \zeta^{2}\right. \\
& \left.+\alpha_{3}\left(R_{\mathrm{eq}}-\rho\right)^{3}+\alpha_{4}\left(R_{\mathrm{eq}}-\rho\right) \zeta^{2}\right] \\
E_{\rho}= & \omega B_{0}\left[\beta_{0} \zeta+\beta_{1}\left(R_{\mathrm{eq}}-\rho\right) \zeta+\beta_{2}\left(R_{\mathrm{eq}}-\rho\right)^{2} \zeta+\beta_{3} \zeta^{3}\right] \\
B_{\varphi}= & B_{0}\left[1+a\left(R_{\mathrm{eq}}-\rho\right)+b\left(R_{\mathrm{eq}}-\rho\right)^{2}+h \zeta^{2}\right. \\
& \left.+q\left(R_{\mathrm{eq}}-\rho\right)^{3}+s\left(R_{\mathrm{eq}}-\rho\right) \zeta^{2}\right] .
\end{aligned}
$$

Not all the terms of expansion with the power less than 3 are presented here because it is taken into account that $E_{\zeta}$ and $B_{\varphi}$ are even functions of $\zeta$, and $E_{\rho}$ is an odd function of this variable.

For calculation of the coefficients $\alpha_{0}, \ldots, \beta_{0}, \ldots$, $a, b, h, \ldots$ we will use Maxwell's equations in the form

$$
\vec{\nabla} \times \vec{E}=-\partial \vec{B} / \partial t, \quad \vec{\nabla} \times \vec{H}=\partial \vec{D} / \partial t, \quad \vec{\nabla} \cdot \vec{E}=0,
$$

with $\vec{B}=\mu_{0} \vec{H}$ and $\vec{D}=\varepsilon_{0} \vec{E}$. We will equate coefficients standing before equal terms of the expansion. For the correct comparison of coefficients, it is necessary that the polynomials for fields have equal powers for $R_{\text {eq }}-\rho$ and $\zeta$ on both sides of the equations. This is why, when substituting fields (1) into the first formula from (2) we should take only linear and quadratic terms for $B_{\varphi}$, and in calculations with the second formula we should take a more precise expansion of $B_{\varphi}$ but only linear and quadratic terms for components of $\vec{E}$.

Substituting Eqs. (1) into (2), we obtain four groups of equations for coefficients of the expansion.

From $[\vec{\nabla} \times \vec{E}]_{\varphi}=-\partial B_{\varphi} / \partial t$ :

$$
\begin{aligned}
\alpha_{0}+\beta_{0}=1, & 2 \alpha_{1}+\beta_{1}=a, \\
3 \alpha_{3}+\beta_{2}=b, & \alpha_{4}+3 \beta_{3}=h .
\end{aligned}
$$

From $[\vec{\nabla} \times \vec{H}]_{\rho}=\partial D_{\rho} / \partial t$ :

$$
-2 h=k^{2} \beta_{0}, \quad-2 s=k^{2} \beta_{1},
$$

where $k=\omega / c, c=1 / \sqrt{\mu_{0} \varepsilon_{0}}$ is the speed of light. 


$$
\text { From }[\vec{\nabla} \times \vec{H}]_{\zeta}=\partial D_{\zeta} / \partial t \text { : }
$$

$$
\begin{aligned}
a & =\frac{1}{R_{\text {eq }},} \\
\frac{1}{R_{\text {eq }}^{2}}+\frac{a}{R_{\text {eq }}}-2 b & =k^{2} \alpha_{0}, \\
\frac{1}{R_{\text {eq }}^{3}}+\frac{a}{R_{\text {eq }}^{2}}+\frac{b}{R_{\text {eq }}}-3 q & =k^{2} \alpha_{1}, \\
\frac{h}{R_{\text {eq }}}-s & =k^{2} \alpha_{2} .
\end{aligned}
$$

From $\vec{\nabla} \cdot \vec{E}=0$ :

$$
\frac{\beta_{0}}{R_{\text {eq }}}-\beta_{1}+2 \alpha_{2}=0, \quad \frac{\beta_{0}}{R_{\text {eq }}^{2}}+\frac{\beta_{1}}{R_{\text {eq }}}-2 \beta_{2}+2 \alpha_{4}=0 .
$$

The equation for $\vec{\nabla} \cdot \vec{H}$ is not included in (2) because the only nonzero component, $H_{\varphi}$, does not depend on $\varphi$ and the equation turns into identity. The equation for $\vec{\nabla} \times \vec{E}_{\zeta}$ also gives the trivial result.

Now we have 12 equations and 14 unknowns.

An additional consideration, that the vector $\vec{E}$ is normal to the surface, can be used, Fig. 3. It gives

$$
2 \beta_{0}=\alpha_{0}+2 R_{c} \alpha_{2} .
$$

Equation (7) can be derived from the equality

$$
\frac{E_{\zeta}}{E_{\rho}}=\frac{\zeta}{\rho-R_{\mathrm{eq}}+R_{c}}
$$

for the points on the surface:

$$
R_{\mathrm{eq}}-\rho \approx \zeta^{2} / 2 R_{c}
$$

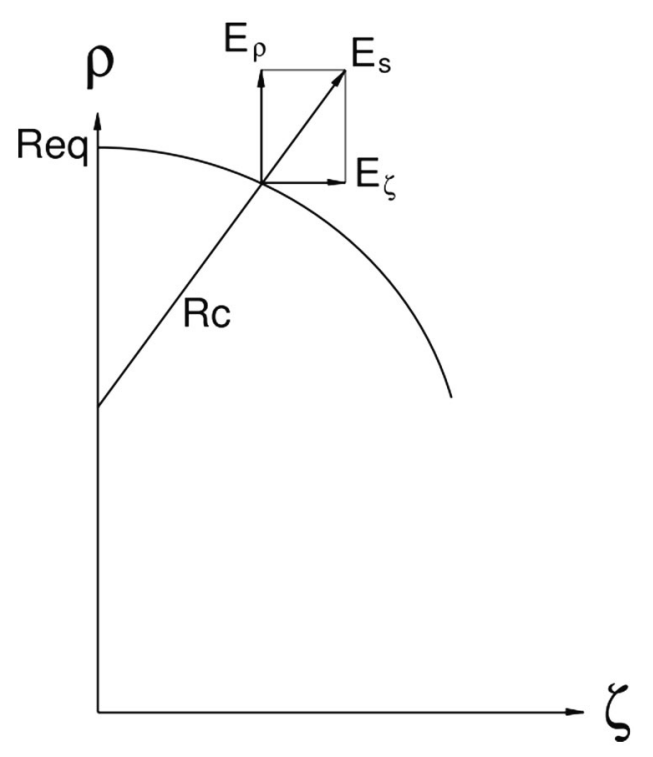

FIG. 3. $E_{s}$ is normal to the surface.
$E_{\zeta}$ and $E_{\rho}$ should be taken from (1), and the lowest power of $\zeta$ is used in (8).

As it will be shown in the next section, fields near the equator do not depend on the local geometry only. So we are forced to introduce some "external" coefficient. Let us analyze the field $B_{\varphi}$ on the border of the cavity. This value is almost constant, but we will have a possibility to compare our results with the fields in a spherical cavity if we take into account the quadratic term:

$$
B_{\varphi}=B_{0}\left(1-\nu \zeta^{2} / 2 R_{c}^{2}\right),
$$

here $\zeta$ is measured for the points on the profile line (9), so that for a spherical cavity $\nu=1\left(B_{\varphi} \sim \sin \theta, \theta \approx \pi / 2\right.$; here $\theta$ is polar angle in the spherical coordinate system). The factor $\nu$ shows the degree of deviation from the constant field value along the cavity profile line. Using this equation together with the third one from (1) under the condition $\left(\rho-R_{\mathrm{eq}}+R_{c}\right)^{2}+\zeta^{2}=R_{c}^{2}$, we obtain for the points on the profile line,

$$
a+2 R_{c} h+\nu / R_{c}=0 .
$$

Now we have 14 equations. Unfortunately, the last equation in (5) turns out to be a linear combination of others and not all the unknowns can be determined. The coefficients for the third powers in the expansions (1) for $E_{\zeta}$ and $E_{\rho}$ : $\alpha_{3}, \alpha_{4}, \beta_{2}$, and $\beta_{3}$, appear undetermined. So, we are forced to limit accuracy of the expansion for the electric field by the second power of decrements and take these coefficients equal to 0 . We will rewrite (1) in a shorter form:

$$
\begin{aligned}
E_{\zeta}= & \omega B_{0}\left[\alpha_{0}\left(R_{\mathrm{eq}}-\rho\right)+\alpha_{1}\left(R_{\mathrm{eq}}-\rho\right)^{2}+\alpha_{2} \zeta^{2}\right], \\
E_{\rho}= & \omega B_{0}\left[\beta_{0} \zeta+\beta_{1}\left(R_{\mathrm{eq}}-\rho\right) \zeta\right], \\
B_{\varphi}= & B_{0}\left[1+a\left(R_{\mathrm{eq}}-\rho\right)+b\left(R_{\mathrm{eq}}-\rho\right)^{2}+h \zeta^{2}\right. \\
& \left.+q\left(R_{\mathrm{eq}}-\rho\right)^{3}+s\left(R_{\mathrm{eq}}-\rho\right) \zeta^{2}\right] .
\end{aligned}
$$

Let us write the chain of equations to determine the rest of coefficients, so that each next coefficient can be calculated using the previous ones (explicit expressions are too cumbersome):

$$
\begin{aligned}
a & =1 / R_{\text {eq }}, \quad h=-a / 2 R_{c}-\nu / 2 R_{c}^{2}, \quad \beta_{0}=-2 h / k^{2}, \\
\alpha_{0} & =1-\beta_{0}, \quad \alpha_{2}=\left(2 \beta_{0}-\alpha_{0}\right) / 2 R_{c}, \\
\beta_{1} & =2 \alpha_{2}+\beta_{0} / R_{\text {eq }}, \quad s=-k^{2} \beta_{1} / 2, \\
b & =1 / R_{\text {eq }}^{2}-k^{2} \alpha_{0} / 2, \quad \alpha_{1}=\left(a-\beta_{1}\right) / 2, \\
q & =\left(2 / R_{\text {eq }}^{3}+b / R_{\text {eq }}-k^{2} \alpha_{1}\right) / 3 .
\end{aligned}
$$

The values of $\alpha_{0}$ and $\beta_{0}$ that will be needed further can be also taken directly from the field calculations. To check the validity of the proposed approach, two cavities were analyzed: fields for the TESLA [4] inner cell were calculated using SLANS [5], fields for the fundamental mode of the spherical cavity (radius $100 \mathrm{~mm}, f=1309 \mathrm{MHz}$ ) were 

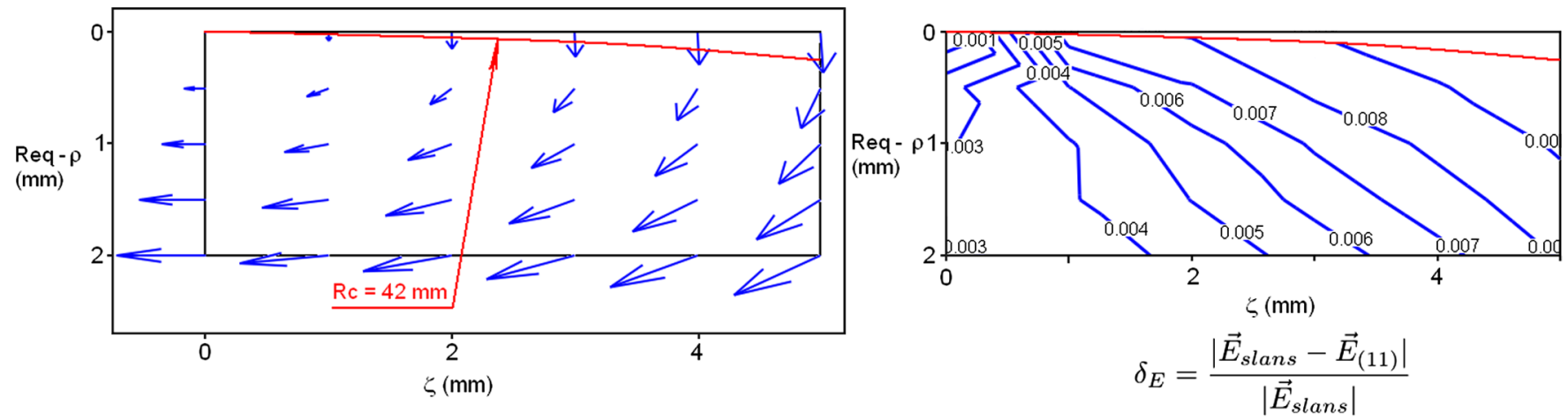

FIG. 4. Left: Electric field in the TESLA cavity near the equator. Vectors outside the cavity volume are formal extrapolation with formula (11). Right: Difference between results calculated with SLANS and with formula (11).
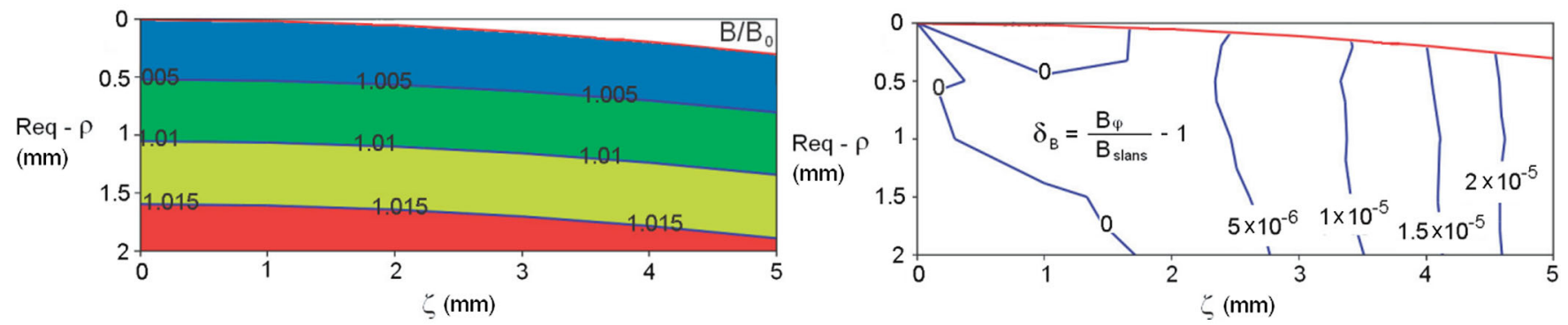

FIG. 5. Left: Magnetic field in the TESLA cavity near the equator. Right: Difference between results calculated with SLANS and with formula (11).

found both analytically (see, for example [6]) and using SLANS. Then solutions for both geometries were found using the proposed expansion in the area with $|z| \leq$ $5 \mathrm{~mm}$ and $y \leq 2 \mathrm{~mm}$. For better accuracy, the mesh $100 \times$ 100 was used; time of calculation was about 10 seconds (computer with $3 \mathrm{GHz}, 3.5 \mathrm{~GB}$ of RAM).

The differences between the solutions for the TESLA cell are (Figs. 4 and 5)

$$
\delta_{E}=\frac{\left|\vec{E}_{\text {slans }}-\vec{E}_{(11)}\right|}{\left|\vec{E}_{\text {slans }}\right|}<1 \%, \quad \delta_{B}=\frac{B_{(11)}}{B_{\text {slans }}}-1<0.0025 \%,
$$

where $\vec{E}_{(11)}$ and $\vec{B}_{(11)}$ are taken from (11).

The magnetic field differs from a constant by about $1 \%$ for $y \leq 1 \mathrm{~mm}$ and less than $2 \%$ for $y \leq 2 \mathrm{~mm}$. If linear terms only are taken for the electric field, the error is less than $1 \%$ for the area $y \leq 1 \mathrm{~mm},|z|<2 \mathrm{~mm}$.

The differences-between the exact analytical solution and SLANS-for the spherical cavity are $<0.3 \%$ for $\vec{E}$ and $<0.0005 \%$ for $B_{\varphi}$. Actually, the value of magnetic field in this cavity is brought out from SLANS with an accuracy of six significant digits, so all of them are correct in the chosen area. The accuracy of the formula (11) - difference between the exact solution for the magnetic field-is even 1 order of magnitude better.

In the next parts of this paper it will be sufficient to use linear only terms of expansion for the electric field and the magnetic field will be considered as a constant. However, the results of the expansion can be used for checking the accuracy of the field calculations by codes and, possibly, in other applications.

\section{DEPENDENCE OF THE UPPER ARC FIELDS ON THE LOWER ARC GEOMETRY}

In construction of the fields near the equator, on the upper arc, we assumed that the field behavior depends mainly on the local geometry. However, this assumption does not hold as shown in Figs. 6 and 7.

One of these cavities has the shape of the inner TESLA cell [4] with the magnetic wall at the iris, another one is described by an elliptic arc with half axes $A=73.856 \mathrm{~mm}$ (along the $Z$ axis) and $B=129.874 \mathrm{~mm}$. Both cavities have the frequency $1300 \mathrm{MHz}$, equatorial radius $R_{\mathrm{eq}}=$ $102.354 \mathrm{~mm}$, and curvature radius $R_{c}=42 \mathrm{~mm}$.

The magnetic field on the initial part of the surface is shown in Fig. 7. Also are shown the parabolic approximations of these fields in the form

$$
y(Z)=H_{1} \cdot\left[1-\nu Z^{2} /\left(2 R_{c}^{2}\right)\right] .
$$

Normalization to $2 R_{c}^{2}$ is used for a comparison with the spherical cavity which has $\nu=1$.

We can conclude that the behavior of fields on the upper arc depends not on geometry of this arc only but also on the 


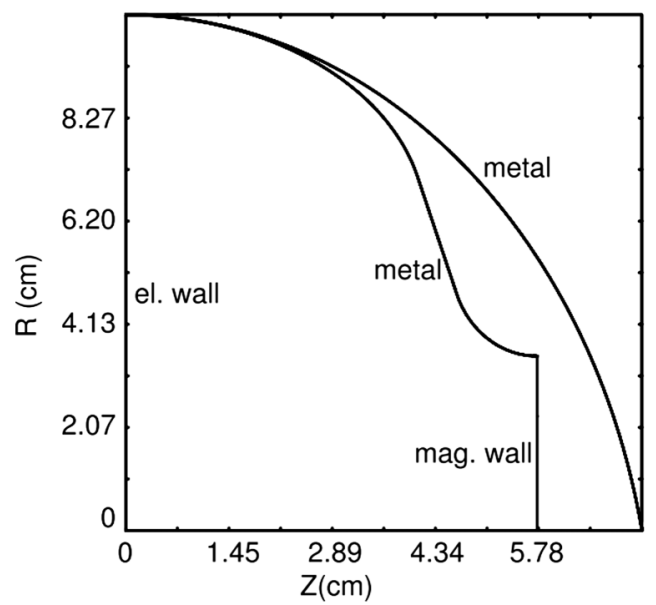

FIG. 6. Example of two cavities with equal frequencies, equal $R_{\text {eq }}$, equal radii of curvature ... (see Fig. 7).

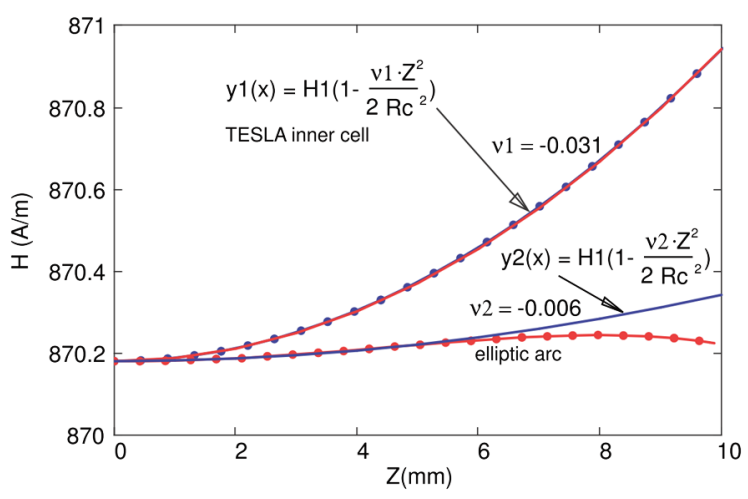

FIG. 7. (See Fig. 6) ... but different behavior of fields near the equator.

geometry of the iris area. In particular, it can be impossible to make a flat field $(\nu=0)$ on the upper arc changing this arc only.

\section{EQUATIONS OF MOTION}

The system of coordinates, fields in the equatorial area, and a rough sketch of a trajectory of an electron in the cavity are shown in Fig. 2. Let us present the magnetic and electric field in this region in the form

$B_{z}=-B_{0} \cos \theta, \quad E_{x}=\alpha y \sin \theta, \quad E_{y}=-\beta x \sin \theta$.

Here $\theta=\omega t, t$ is time, and $\alpha$ and $\beta$ are coefficients of proportionality. We will use $\alpha=\alpha_{0} \omega B_{0}$ and $\beta=\beta_{0} \omega B_{0}$, taking $\alpha_{0}$ and $\beta_{0}$ from (12). Adding of higher terms of the expansions has an insignificant effect on the results. However, the coefficient $h$ describing dependence of the magnetic field on $\zeta^{2}$ should be calculated because it defines values of $\alpha_{0}$ and $\beta_{0}$.

Now we can write the equations of motion taking into account these three components of fields:

$$
e\left(E_{x}+\dot{y} B_{z}\right)=m \ddot{x}, \quad e\left(E_{y}-\dot{x} B_{z}\right)=m \ddot{y} .
$$

The charge of electron is taken positive to simplify writing. Dots designate derivatives with respect to time. Replacing derivatives with respect to time by derivatives with respect to the phase angle $\theta$ (designated by primes), so that

$$
\dot{x}=\omega x^{\prime}, \quad \ddot{x}=\omega^{2} x^{\prime \prime}, \quad \text { and so on, }
$$

one can obtain the equations of motion in a normalized form:

$$
\begin{aligned}
& x^{\prime \prime}=M\left[(1-p) y \sin \theta-y^{\prime} \cos \theta\right], \\
& y^{\prime \prime}=M\left(-p x \sin \theta+x^{\prime} \cos \theta\right) .
\end{aligned}
$$

Here $M=e B_{0} / m \omega$ is the magnetic parameter of the motion, and $p=\beta /(\alpha+\beta)=\beta_{0}$ can be named the geometrical parameter depending on the curvature of the surface near the equator and some inner geometrical details (value of $\nu$ ) but not on the field amplitudes: $p=$ $\left(d E_{n} / d x\right) / \omega B_{0}$, where $E_{n}$ is the normal to the surface electric field at the area of multipacting. From (12), one can obtain

$$
p=\left(c^{2} / \omega^{2}\right) \cdot\left(a / R_{c}+\nu / R_{c}^{2}\right) .
$$

The set (15) was solved with MATHCAD by the method of Runge-Kutta.

\section{CONDITION OF STABILITY}

In the case when multipacting occurs near the cavity equator, the starting electron should be described both by the phase of the field and the distance from the equator because the electric field $E_{y}$ changes with this distance $x$, see (13). If the electron has not an equilibrium initial phase and position, after the flight to the next impinge onto the surface, its phase and position will change:

$$
\begin{aligned}
& \Delta \theta_{2}=\left(\partial \theta_{2} / \partial \theta_{1}\right) \Delta \theta_{1}+\left(\partial \theta_{2} / \partial x_{1}\right) \Delta x_{1}, \\
& \Delta x_{2}=\left(\partial x_{2} / \partial \theta_{1}\right) \Delta \theta_{1}+\left(\partial x_{2} / \partial x_{1}\right) \Delta x_{1},
\end{aligned}
$$

where $\Delta \theta_{1}$ and $\Delta x_{1}$ are deviations from the equilibrium phase and position, respectively, at the start point, $\Delta \theta_{2}$ and $\Delta x_{2}$ are deviations after the flight. The derivatives in (17) are taken for the equilibrium situation when the time of flight is equal to the integer odd number of half periods:

$$
\theta_{2}-\theta_{1}=(2 n-1) \pi
$$

The system (17) can be written as a matrix product:

$$
\left(\begin{array}{l}
\Delta \theta_{2} \\
\Delta x_{2}
\end{array}\right)=\left(\begin{array}{ll}
a & b \\
c & d
\end{array}\right)\left(\begin{array}{l}
\Delta \theta_{1} \\
\Delta x_{1}
\end{array}\right) \equiv A\left(\begin{array}{c}
\Delta \theta_{1} \\
\Delta x_{1}
\end{array}\right) .
$$

After the $N$ th flight, the deviations are

$$
\left(\begin{array}{c}
\Delta \theta_{N+1} \\
\Delta x_{N+1}
\end{array}\right)=A^{N}\left(\begin{array}{c}
\Delta \theta_{1} \\
\Delta x_{1}
\end{array}\right) .
$$


So, for stability, it is necessary that

$$
\lim _{N \rightarrow \infty} A^{N}=0,
$$

and this requirement is fulfilled if the characteristic roots of the matrix equation

$$
A-\lambda I=0,
$$

where $I$ is a unitary matrix, meet the conditions

$$
\left|\lambda_{1}\right|<1, \quad\left|\lambda_{2}\right|<1 .
$$

The derivatives for (17) were found numerically solving the equations of motion (15) for different $p$ and $M$, and substituted into Eq. (18).

\section{MULTIPACTING MAPS}

The areas where the conditions (19) are met are shown in Figs. 8 and 9; impact energy of electrons is also shown. The "boundary of stability" is defined as the locus of points where either $\left|\lambda_{1}\right|=1,\left|\lambda_{2}\right|<1$ or $\left|\lambda_{1}\right|<1$, $\left|\lambda_{2}\right|=1$. The emission energy of electrons is taken equal to $2 \mathrm{eV}$.

The multipacting area is limited by the line where the energy of the impact electrons is high enough to produce the secondary emission yield SEY $>1$.

The very important role of the emission energy of the secondary electrons should be emphasized: increase of this energy leads to the same increase of the impact energy of the electrons. Some authors use for calculations $4 \mathrm{eV}$ as the emission energy. In this case all the data for the equal energy curves in Fig. 8 and 9 should be doubled.
This fact follows from the homogeneity of the equations of motion (15): if all the functions- $x, y$, and their derivatives - are multiplied by a constant, the equation keeps true. The sizes of trajectories as well as velocities and accelerations at each point of the trajectory increase $\sqrt{2}$ times if the emission energy is doubled.

This feature is not inherent in, say, multipacting in a flat gap.

Parameters $M$ and $p$ are dimensionless. However, the energy on these maps is in electron-volts because actually we calculate the ratio of start and impact velocities, and give a value for the start velocity.

One more important feature of multipacting in crossed rf fields is the angle the electrons impinge the surface. Dependence of the secondary emission yield on the angle of incidence according to [7] is

$$
\delta=\delta_{0} e^{\kappa(1-\cos \theta)},
$$

where $\delta_{0}$ is the SEY for the electrons falling normally to the surface, the angle $\theta$ is measured from this normal, and the coefficient $\kappa$ can be found from the data presented in [7]. Though this coefficient is not known for niobium, we can roughly estimate it knowing that it changes from 1.25 for aluminum to 0.75 for molybdenum. This coefficient is close to 1 for copper. If we accept this value $\kappa=1$ for niobium, we will have an increase $\delta / \delta_{0}=1.08$ for the point $M=2, p=0.3$, where $\theta=22.5^{\circ}$. This increase grows fast with the angle, so for $\theta=60^{\circ}$ this increase is $65 \%$. The angle of incidence for different points of the multipacting zones is shown in Fig. 10-for the first order, and in Fig. 11-for the second order of this two-point multipacting. The shapes of trajectories are shown for

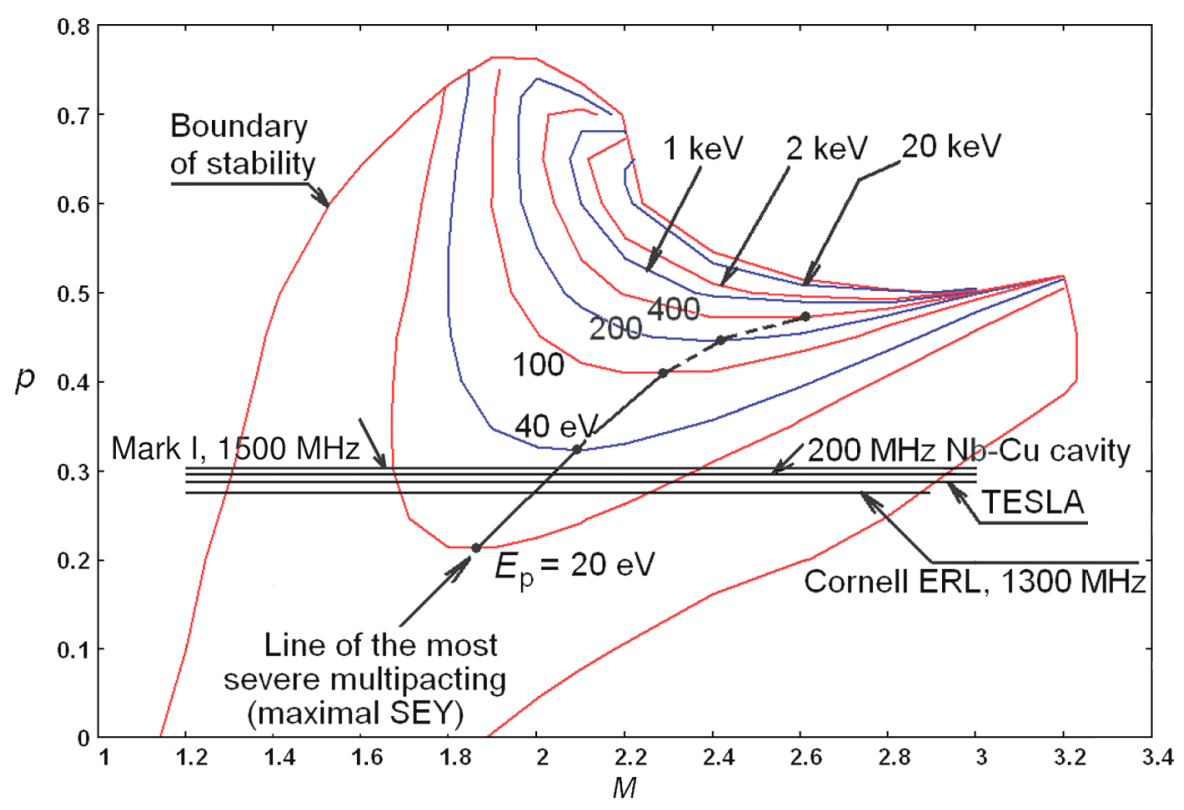

FIG. 8. Equatorial cross-field two-point first-order multipacting zone. Emission energy of the electrons is $2 \mathrm{eV}$. Examples for several geometries: 1-200 MHz Nb-Cu cavity [8], 2-TESLA cavity [4], 3-Cornell ERL cavity [9], Mark I cavity [10]. $E_{p}$ is the impact energy. 


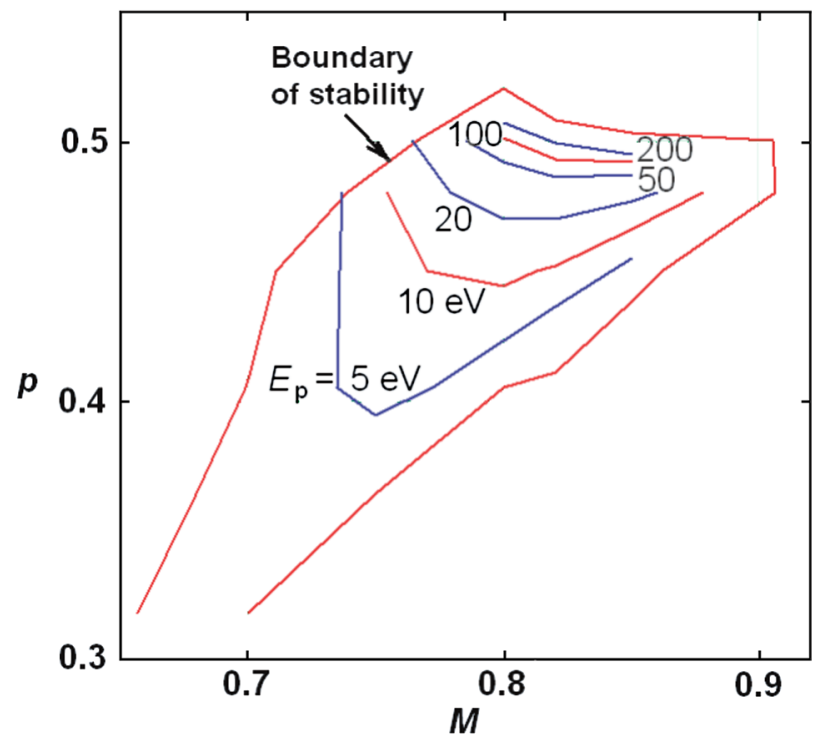

FIG. 9. Equatorial cross-field two-point second-order multipacting zone.

several points of the area of stable resonant motion. Maximal impact energy of electrons for each value of $p$ is reached on the line connecting the lowest points of the lines of constant $E_{p}$, see Fig. 8. Because of the increase of the angle with $M$, as can be seen from the graphs, the SEY will also increase and the line of the most severe multipacting will be shifted to higher values of $M$ from these lowest points of the curves of constant energy (Fig. 8) and

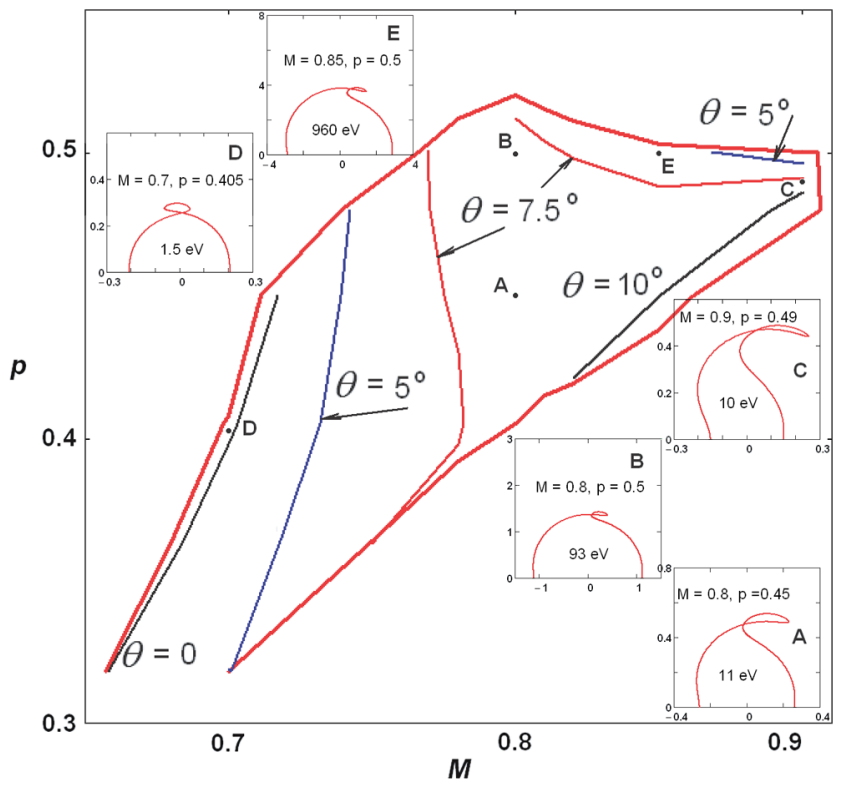

FIG. 11. Incidence angle of the electrons in the second zone of the two-point multipactor. See explanations in Fig. 10.

SEY can be higher than 1 even though for the normal incidence it can be less than 1 .

The incidence angle for the second-order multipacting does not vary in so wide limits as for the first order; however, the energy can also reach the values big enough for ignition of the multipactor discharge. The value of $p$ should be big enough for this case.

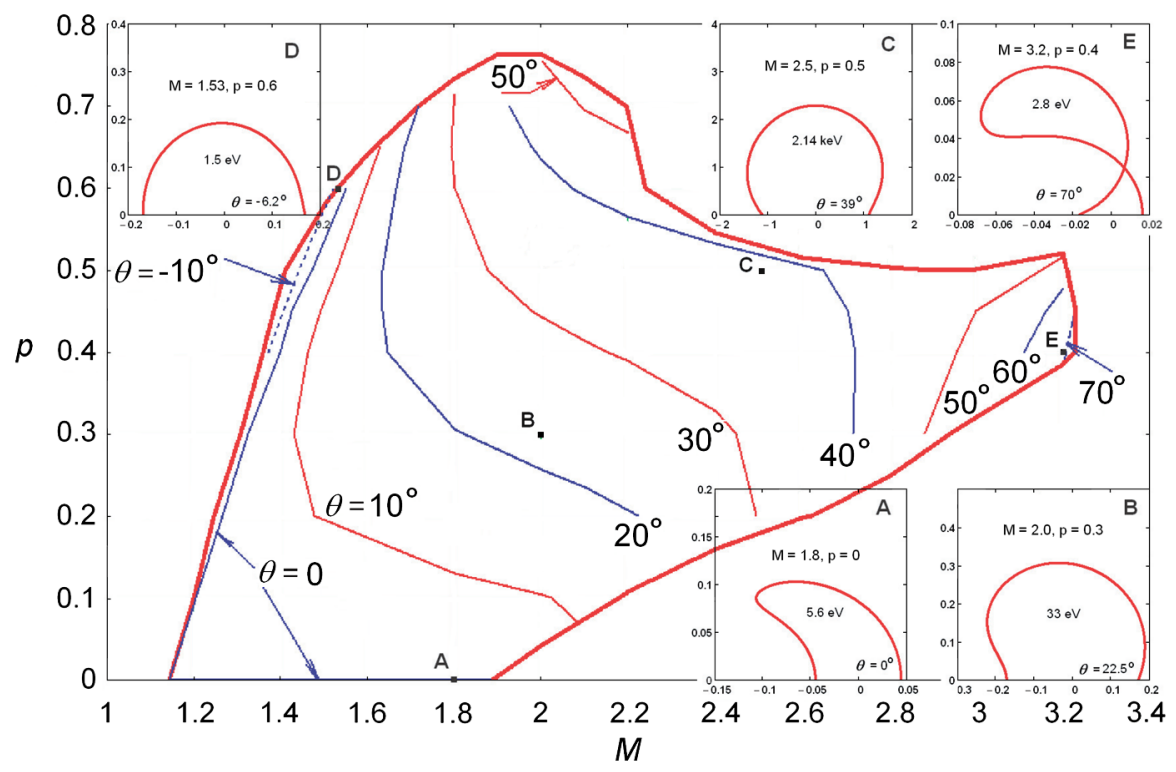

FIG. 10. Incidence angle of the impacting electrons in the first zone of the two-point multipactor. The initial velocity vector is normal to the surface. Shapes of trajectories are shown for several points in the area of stability. Dimensions of trajectories-in millimeters. Trajectories are shown in different scales but equal for both axes to show the angle. 


\section{DEVIATIONS FROM THE ELLIPTIC GEOMETRY}

One can see from Figs. 8-11 a strong dependence of the multipacting conditions on the geometrical parameter $p$. The geometry of the surface near the equator can be not ideally elliptic and the dimensions of the trajectory are very small, so even small deformations, compared to the size of the cavity, can change significantly the geometrical conditions. The equator of the cavity is usually a place where two half cells are welded together, and the weld seam can be machined and chemically processed to remove the roughnesses, or a bead, though smoothed, can be left at the line of the equator.

In the case when the equatorial region is machined flat, usually before the welding, and if the welding does not change this flatness too much, the change of the parameter $p$ is insignificant. The geometry with the flat equator is shown in Fig. 12. The pattern of the electric field is practically the same as for the original shape without the flatness, see Fig. 4. The value of $p$ is not zero as could be expected from (16) for $R_{c}=\infty$ in the case of the flatness. Apparently the size of the flatness is not big enough. The force lines reflect the ratio between $\alpha$ and $\beta$ from (13),

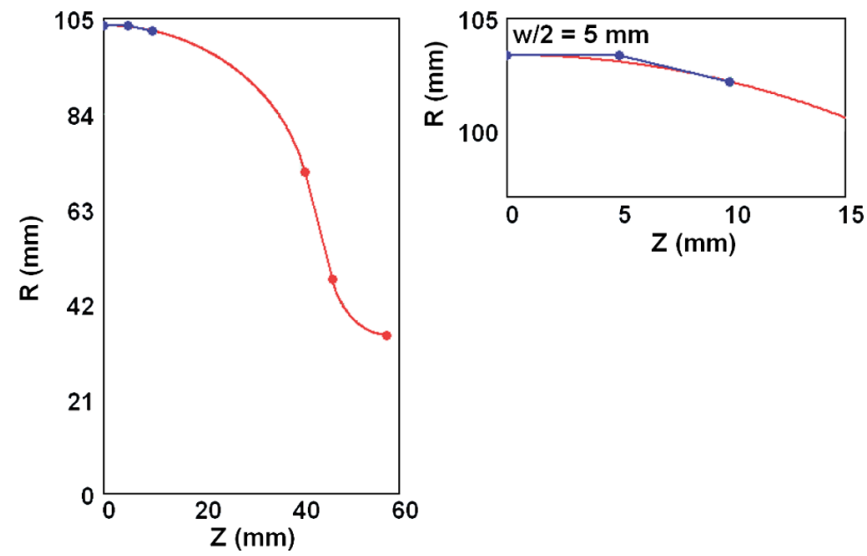

FIG. 12. Flat equator in the case of the TESLA cavity (blue line). because they have a shape of ellipse with the ratio of axis equal to $\sqrt{\beta / \alpha}$ as follows from

$$
\frac{E_{y}}{E_{x}}=\frac{d y}{d x}=\frac{-\beta x}{\alpha y} .
$$

The SLANS calculations for $\alpha$ and $\beta$ show that $p=$ $\beta /(\alpha+\beta)$ changes from $p=0.29$ for the original shape to $p=0.26$ for the width of the flat region $w=10 \mathrm{~mm}$. So, the flatness of the equator does not change the value of this parameter significantly, and if it changes, makes it smaller, hampering the origin of multipacting. (We will not discuss here very large flat regions which can lead to a one-point multipactor [11].)

In the case of a bead along the equator, the picture can change substantially.

To understand what is the real shape of the weld seams, the measurements of replica moulds were done with help of Coordinate Measuring Machine ACCURA ${ }^{\mathrm{TM}}$ from Carl Zeiss. The replica moulds were earlier prepared for studying the defects on the cavity surface near the equator, not specifically for the seam studying, so not the whole seam can be seen in all the cases. The replicas were made for the Cornell ERL main linac cavity, and for two TESLA cavities - made by Advanced Energy Systems, Inc. (AES) Medford, NY, and by ACCEL Instruments GmbH, Bergish Gladbach, Germany. Results of measurements are presented in Fig. 13. All the seams are shown in the same scale (but about 10 times larger in the radial direction) for easier comparison. Limits of the seams are arbitrarily shown by red circles. Points outside these limits (not all of them are shown) were used for rms fitting the cavity profile to the ideal circular shape, shown in red. The radius of this circle was taken equal to the radius of curvature at the equator, $48 \mathrm{~mm}$ in the case of the ERL cavity, for TESLA it is a circle, $R=42 \mathrm{~mm}$.

Comparison of sizes of the weld seams and of the trajectories at the most probable multipacting conditions (points B on both Figs. 10 and 11) shows that the size of seam (5-10 $\mathrm{mm}$ in the $Z$ direction) is several times larger than the typical size of the orbit $(0.5-1 \mathrm{~mm})$. The curvature of the surface defining the value of $p$ (16) changes within
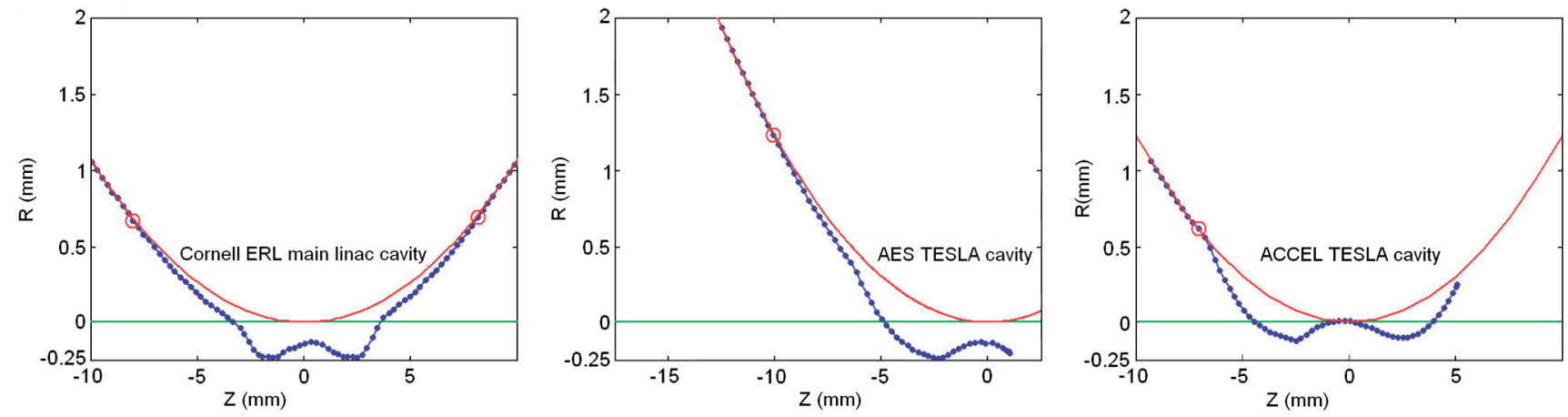

FIG. 13. Weld seam profiles for the cavities made by Cornell, AES, and ACCEL. 

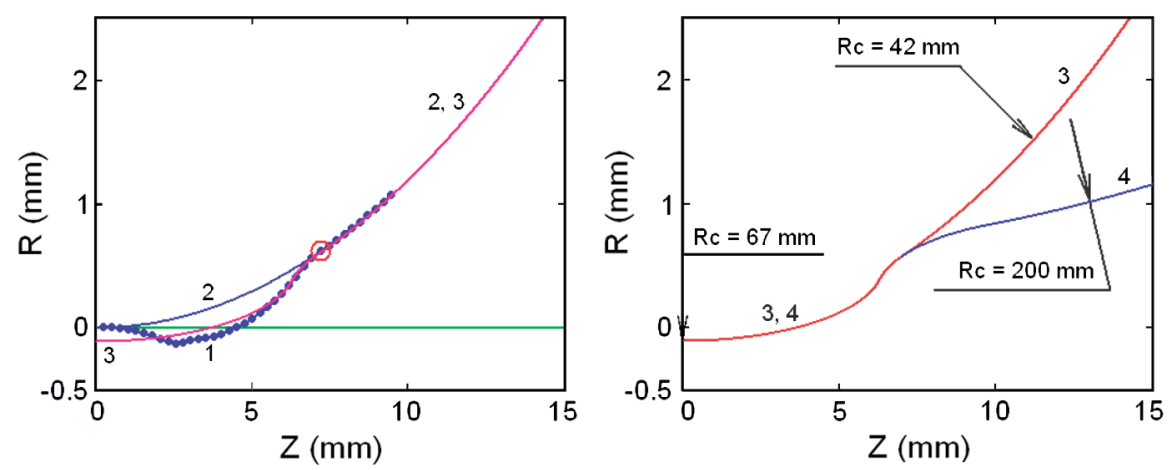

FIG. 14. Simplified approximation of the cavity seam profile for the TESLA cavity made by ACCEL and for the $200 \mathrm{MHz} \mathrm{Nb}-\mathrm{Cu}$ cavity fabricated at CERN and welded by ACCEL. 1-Measured profile of the TESLA cavity; 2-ideal contour with radius of 42 mm; 3 -approximation of the TESLA seam profile by two ellipses; 4-approximation of the $200 \mathrm{MHz}$ cavity seam profile.

this orbit as compared to the design value. So, the seam can have a dramatic effect on the multipacting emergence.

The SLANS calculation with the shape close to the shape of the TESLA cavity made by ACCEL, see Fig. 13, with the equator seam area approximated by four conjugated circular arcs (for the half-cell), gave the value of $p$ close to that of the ideal shape, $p=0.27$.

Existence of the second-order multipacting in the $200 \mathrm{MHz} \mathrm{Nb-Cu}$ cavity [8], and its absence in the $1300 \mathrm{MHz}$ cavities, caused us to compare the influence of the seam on the multipacting zones in these cavities. Regretfully, the shape of the seam in the $200 \mathrm{MHz}$ cavity is not available at the moment but we can make the most general assumptions.

Since the central bead of the seam has an insignificant effect on the geometrical parameter $p$, for further calculations of the fields the shape of the seam was simplified as it is shown in Fig. 14. The seam area was described by two elliptic arcs conjugated to one another and to the main circle of the equatorial area. The seam size should not depend too much on the cavity size, both cavities were welded by the same producer, so for the $200 \mathrm{MHz}$ cavity the same seam ellipses parameters were used. [As it was commented by Geng [12] "The copper cavity (before $\mathrm{Nb}$ film deposition-VS) was electron beam welded from both inside and outside. This practice was sometimes also used for making niobium cavities. The general tendency is that this results in a smaller under-bead (because of less heat deposition during each of the two passes of the electron beam). This in turn means that there is a high probability that there is little or no "bump" at center of equator" (at $Z=0$ in profiles in Fig. 13). So, there is some uncertainty in our considerations.] As it is shown in the figure, the radius of curvature of the seam at the lowest point is bigger than the radius of the ideal shape for the TESLA cavity and smaller than the $200 \mathrm{MHz}$ ideal radius. In the next section it will be shown that this fact can explain the existence of the second-order multipacting in the $200 \mathrm{MHz}$ cavity and its absence in the TESLA cavity.

\section{COMPARISON WITH EXPERIMENT}

Any given cavity has its own value of $p$. The most severe multipacting (if any) should be expected at the value of $M$ corresponding to the crossing of the line of maximal SEY and the line of the given $p$. Five examples of cavities with calculated and observed values of multipacting fields are presented in Table I.

One can see good agreement between calculated and observed fields. Some difference can be explained by the fact that the discharge starts below the most sensitive (calculated) point and terminates above it. This is especially true for big values of $p$.

Calculations with the shape of the seam in the $200 \mathrm{MHz}$ cavity shown in Fig. 14 give for the parameter $p$ the value of 0.35 , instead of 0.296 for the ideal shape. According to

TABLE I. Parameters of some cavities and fields of calculated and observed multipacting.

\begin{tabular}{|c|c|c|c|c|c|c|c|}
\hline & $R_{\mathrm{eq}}$ & $R_{c}$ & $\nu$ & $p$ & $M$ & $\begin{array}{c}E_{\mathrm{acc}}, \mathrm{MV} / \mathrm{m}, \\
\text { calculated }\end{array}$ & $\begin{array}{c}E_{\mathrm{acc}}, \mathrm{MV} / \mathrm{m}, \\
\text { observed }\end{array}$ \\
\hline $\mathrm{Nb}-\mathrm{Cu}$ cavity [8], $200 \mathrm{MHz}$ & 685 & 200 & -0.0841 & $\begin{aligned} & 0.296 \\
\approx & 0.5(?)\end{aligned}$ & $\begin{array}{l}2.03 \\
0.78\end{array}$ & $\begin{array}{l}3.3 \\
1.3\end{array}$ & $\begin{array}{c}2.8-4 \\
1\end{array}$ \\
\hline TESLA $1300 \mathrm{MHz}[13,14]$ & 103.4 & 42 & -0.0313 & 0.286 & 2.0 & 22 & $20,17-20$ \\
\hline TRISTAN $508 \mathrm{MHz}$ [13] & 258.7 & 98.9 & $\mathrm{n} / \mathrm{a}$ & $\mathrm{n} / \mathrm{a}$ & $\sim 2$ & 8.5 & 7 \\
\hline Cornell ERL [9], $1300 \mathrm{MHz}$ & 102.9 & 48.1 & 0.0068 & 0.276 & 2.0 & 22 & Not observed up to $27 \mathrm{MV} / \mathrm{m}$ \\
\hline Mark I, $1500 \mathrm{MHz}$ [10] & 92.02 & 29.63 & -0.0618 & 0.303 & $1.44(2.05)$ & $17.6(25.0)$ & 17.6 limited by $21 \mathrm{MV} / \mathrm{m}$ \\
\hline
\end{tabular}


Fig. 9, for the second-order multipacting the needed value of $p$ should be about 0.5 . It is possible that the seam is even deeper in this cavity. Let us remind that this seam was done from inside contrary to the usual practice of the outside welding of the equator of smaller cavities, and as it is said in [8] further mechanical smoothing has been done by grinding locally all the sharp points of the internal surface.

The author does not have enough information about the TRISTAN cavity geometry, so it was supposed that the value of $M$ does not differ too much from the values for other cavities $(M \approx 2)$.

Multipacting was not observed in the Cornell ERL cavity up to $E_{\text {acc }}=27 \mathrm{MV} / \mathrm{m}$. Let us note that this cavity has a minimal value of $p$ between others in the table, and so the minimal energy of impacting electrons compared to other cavities in the list. This can be a reason of absence of a detectable multipacting in this cavity.

A detailed study of multipacting was done by Knobloch et al. [10]. Results of simulations presented in this work for the cavity Mark I (in which a repetitive multipacting was observed) give the same starting value as can be obtained from Fig. $8\left(E_{p k}=32 \mathrm{MV} / \mathrm{m}\right.$, or $E_{\text {acc }}=17.6 \mathrm{MV} / \mathrm{m}$, $M=1.45)$, if we accept the same values of $E_{s}(3 \mathrm{eV})$ and $\mathrm{SEY}=1$ at $E_{p}=20 \mathrm{eV}$ as in this paper. Apparently, the most severe multipacting was not reached because the maximal reached value of $E_{p k}$ was $38 \mathrm{MV} / \mathrm{m}$ $\left(E_{\text {acc }}=21 \mathrm{MV} / \mathrm{m}\right)$. The author refers to field emission and related thermal breakdown which prevented from reaching higher fields. However, it was expected "that multipacting would have been active up to about $42.5 \mathrm{MV} / \mathrm{m}$ if the cavity had not been limited by thermal breakdown." According to Fig. 8, multipacting in this geometry $(p=0.305)$ would exist at even higher fields having the maximum at $M=2.05$ that corresponds to $E_{p k}=45.5 \mathrm{MV} / \mathrm{m}$. The early start-up of the multipacting with $E_{p}$ as small as $20 \mathrm{eV}$ is unlikely but it can be explained if the actual $p$ was bigger than calculated because of the shape deviation from the ideal geometry due to the seam or other defects of the surface. In this case, the value of $E_{p}$ will be bigger at the same start-up field. Increase of $\delta / \delta_{0}$ about $8 \%$ (see above) or more, because of uneven surface, due to the incidence angle also works for explanation of $E_{p}$ as low as this.

Multipacting in this experiment was observed "at several distinct areas in the equator region" though it constantly shifted "to different places of higher SEY around the equator" after desorption of surface adsorbates. We can suppose that not only quality of the surface but also the shape deviations can be a cause of multipacting at these distinct areas.

Energy of impacting electrons about $30 \mathrm{eV}$ (as in Fig. 8) is low enough to have the secondary emission yield SEY $>1$. This energy is found for $E_{s}=2 \mathrm{eV}$. If we take as a possible the value of $E_{s}=4 \mathrm{eV}$ the values of $E_{p}$ on Figs. 8 and 9 should be doubled. Possibly, the truth is somewhere between. Besides, an increase of the SEY due to the nonzero angle of incidence should be also taken into account; see Figs. 10 and 11. Since the phenomenon is very sensitive to the value of $E_{s}$, it is desirable to have more reliable information about the emission energy of electrons for niobium.

Processing of the multipactor in a cavity may consist not only in lowering the secondary emission yield but also in lowering the starting energy of the secondary electrons. As it was noted above, the energy of the impacting electrons will also decrease in this case and lead to a lower SEY.

The formula for the magnetic parameter $M=e B_{0} / m \omega$ can be rewritten in the form

$$
B_{0}[\mathrm{mT}]=35.7 M \cdot f[\mathrm{GHz}],
$$

where limits of $M$ depend on the value of $p$. Cavities with the same frequency can have different regions of multipacting having different geometry. This formula is similar to experimental formulas $[13,15,16]$ but now it gives a clear physical explanation.

Comparing the formula (20) with the phenomenological formulas from the cited papers, one can find the corresponding values of the parameter $M$ as it is shown in Table II. The first formula by Fabbricatore et al. follows from a simple assumption that the frequency of the multipacting motion is 2 times higher than the frequency of the field oscillations. Actually, it is based on the static magnetic field. The second formula is a correction of the first one by dividing the first value by $\sqrt{2}$. In the third line, there is the value corresponding to an experiment with a $3 \mathrm{GHz}$ cavity.

Cavities of Saito and Geng had different shapes, this can explain the difference in the values of the coefficients before $f$ in Table II. Geng made an attempt to unite different data involving dependences on frequency but apparently the shift from direct proportionality to frequency

TABLE II. Experimental formulas for the multipacting fields and corresponding values of $M$.

\begin{tabular}{llc}
\hline \hline Author & \multicolumn{1}{c}{ Formula } & Value of $M$ \\
\hline Fabbricatore et al. (1995) [16] & $B[\mathrm{mT}]=78 f[\mathrm{GHz}]$ & 2.18 \\
& $B[\mathrm{mT}]=55 f[\mathrm{GHz}]$ & 1.54 \\
& $B[\mathrm{mT}]=57 f[\mathrm{GHz}]$ & 1.60 \\
Saito (2001) [13] & $B[\mathrm{mT}]=60 f[\mathrm{GHz}]$ & 1.68 \\
Geng (2003) [15] & $B[\mathrm{mT}]=5+55 f[\mathrm{GHz}]$ & $1.54+0.14 / f$ \\
\hline \hline
\end{tabular}


was due to different shapes of the cavities, not to their frequencies.

The formula (20) can be rewritten in a more practical form. For any cavity the value $B_{p k} / E_{\text {acc }}$ is known as one of the most important figures of merit. If $B_{0}$ is the magnetic field at the equator and $E_{0}$ is the accelerating field, both corresponding to presence of multipacting, then $B_{p k} / E_{\text {acc }}=B_{0}(1+\varepsilon) / E_{0}$. The value of $\varepsilon$ shows the deviation of the peak field from the field at the equator and for a thoroughly optimized cavity is small. For example, the TESLA cavity has $\varepsilon=0.005$, and the Cornell ERL cavity has $\varepsilon=0$. So, this value can be neglected. From the forgoing expression and from (20), we can obtain the accelerating field of the most probable multipacting:

$$
E_{0}=\frac{B_{0}(1+\varepsilon)}{B_{p k} / E_{\mathrm{acc}}}=\frac{35.7 \cdot M \cdot f}{B_{p k} / E_{\mathrm{acc}}}[\mathrm{MV} / \mathrm{m}, \mathrm{MHz}, \mathrm{mT}] .
$$

The value of $M$ for the most probable multipacting depends on the geometric parameter of the cavity and can be taken, for example, see Fig. 8, equal to 2.0 for $p=$ 0.27 or 2.1 for $p=0.33$.

\section{CONCLUSIONS}

A careful description of multipacting near the cavity equator is presented. Electric and magnetic fields in the equatorial region of an elliptic cavity given in the form of the Taylor series were compared with theory and simulations and showed a very good accuracy of the expansion. Using these analytical approximations, zones of the first and second order of the two-point discharge were found and energy and incident angles maps of both zones are presented. Influence of the equatorial weld seam shape is discussed in detail. The predictions are compared with experimental results. The experimental formulas of the multipacting field obtained a clear physical meaning. Also, a possible explanation of the existence of the second-order zone in the $200 \mathrm{MHz} \mathrm{Nb}-\mathrm{Cu}$ cavity is offered.

The first, short version of this paper was presented at EPAC 2004 [17].

\section{ACKNOWLEDGMENTS}

The paper benefited from numerous discussions with Sergey Belomestnykh and useful remarks made by Rongli Geng. I am thankful to Mingqi Ge for providing the replica moulds for the seam measurements and also to John Kaufman and James Sears for measurements with
CMM and discussions. I am indebted to Nick Valles for attentive reading of the manuscript. Pictures of the seams of the $200 \mathrm{MHz}$ cavity were kindly sent to me by Roberto Losito from CERN. The stimulating interest of Georg Hoffstaetter and of other colleagues from our lab was also helpful. This work was supported by NSF Award No. DMR-0807731.

[1] V. Shemelin, H. Padamsee, and R. L. Geng, Nucl. Instrum. Methods Phys. Res., Sect. A 496, 1 (2003).

[2] P. Ylä-Oijala, J. Lakkarinen, S. Järvenpää, and M. Ukkola, MultiPac 2.1 (Rolf Nevanlinna Institute, Helsinki, 2001).

[3] K. Krebs and H. Meerbach, Ann. Phys. (Berlin) 450, 189 (1955).

[4] B. Aune et al., Phys. Rev. ST Accel. Beams 3, 092001 (2000).

[5] D. Myakishev and V. Yakovlev, in Proceedings of the Particle Accelerator Conference, Dallas, TX, 1995 (IEEE, New York, 1995).

[6] S. Belomestnykh, LEPP Report No. SRF 941208-13, Cornell University, 1994.

[7] H. Bruining, Physics and Applications of Secondary Electron Emission (Pergamon Press, New York, 1954).

[8] R. Geng et al., in Proceedings of the 20th Particle Accelerator Conference, Portland, OR, 2003 (IEEE, New York, 2003), p. 1309.

[9] N. Valles and M. Liepe, in Proceedings of the SRF2009, Berlin, Germany, 2009, p. 538 [http://www.jacow.org].

[10] J. Knobloch, W. Hartung, and H. Padamsee, in Proceedings of the 8th SRF Workshop, 1997, Abano Terme, Padova, Italy [http://www.jacow.org].

[11] U. Klein and D. Proch, in Proceedings of the Conference on Future Possibilities for Electron Accelerators (1979), pp. N1-N17.

[12] R. Geng (private communication).

[13] K. Saito, in Proceedings of the 10th Workshop on RF Superconductivity, 2001, Tsukuba, Japan.

[14] K. Twarowski, L. Lilje, and D. Reschke, in Proceedings of the 11th Workshop on RF Superconductivity, 2003, Lübeck/Travemünde, Germany [http://www.jacow.org].

[15] R. Geng, in Proceedings of the 20th Particle Accelerator Conference, Portland, OR, 2003 (Ref. [8]), p. 264.

[16] P. Fabbricatore, G. Gemme, R. Musenich, R. Parodi, and S. Pittaluga, in Proceedings of the 7th SRF Workshop, 1995, Gif sur Yvette, France [http://www.jacow.org].

[17] V. Shemelin, in Proceedings of the 9th European Particle Accelerator Conference, Lucerne, 2004 (EPSAG, Lucerne, 2004). 